\title{
AUTOCORRELATION EFFECTS IN MANUFACTURING SYSTEMS PERFORMANCE: A SIMULATION ANALYSIS
}

\author{
Diego Crespo Pereira \\ David del Rio Vilas \\ Nadia Rego Monteil \\ Rosa Rios Prado \\ Alejandro Garcia del Valle \\ University of A Coruna \\ C. Mendizabal $\mathrm{s} / \mathrm{n}$ \\ Ferrol (A Coruna) 15403, SPAIN
}

\begin{abstract}
Autocorrelation has been pointed out as one of the most challenging issues in manufacturing systems modeling. Numerical experimentation has shown that it may either enhance or harm performance. Furthermore, there is not yet a general agreement in what a realistic autocorrelation model is or whether it is actually relevant for practical applications. This paper provides a simulation analysis of the effects on performance caused by manufacturing process parameters following autoregressive (AR) processes. AR time series are employed for modeling variations in parameters that happen at a time scale different from the corresponding to process cycle execution. Three basic configurations are analyzed: serial line, assembly process and a disassembly process. A case study from the natural slate tiles industry is presented showing the differences obtained in simulation results between a model in which independent and identically distributed (i.i.d.) assumptions are adopted and one in which autocorrelation effects are considered.
\end{abstract}

\section{INTRODUCTION}

Autocorrelation is defined as the correlation between successive observations of a time series (Banks et al., 2010). For instance, if $x_{t}$ is the observation of the variable $x$ in the time period $t$, then the autocorrelation of lag $l$ is:

$$
\rho_{l}=\frac{\operatorname{cov}\left(x_{t}, x_{t, t-l}\right)}{\operatorname{var}(x)}
$$

The question on how autocorrelation affects manufacturing processes has been addressed in previous works by different authors. Positive autocorrelation in event streams causes a "bursty behavior" in which events seem to be clustered (Altiok and Melamed, 2001). Thus, successive events separated by either short or long time periods tend to be followed by similar corresponding observations Negative autocorrelation, on the other hand, causes an alternation between short and long time periods between events. Autocorrelation effects are present in production lines time series even if arrival processes and service times are assumed i.i.d (independent and identically distributed). In this case, autocorrelation is present in the output processes, which have been studied by authors such as Hendricks and Mcclain (1993). It has been found to explain the response of the system to changes in buffers capacity.

Results from queuing theory show that autocorrelation in service or inter-arrival times may severely affect queue lengths and waiting times (Livny et al. 1993). Manufacturing lines can be modeled as queues 


\section{Crespo Pereira, D. del Rio Vilas, N. Rego Monteil, R. Rios Prado and A. Garcia del Valle}

networks and therefore autocorrelation in process variables may have a relevant effect in performance. Altiok and Melamed (2001) studied the relevance of autocorrelation in different performance metrics for pull type processes. They introduce autocorrelation by means of TES (Transform-Expand-Sample) processes and their results alert on the potential losses on accuracy that might be derived from an inadequate use of independence assumption. Takahashi and Nakamura (1998) studied the case of Just-In-Time systems achieving similar conclusions. Balcioglu et al. (2007) provide an approximate solution for the mean waiting times when autocorrelation is present in time to failure time series. They later proposed an approximate method to analyze the effect of splitting and superposition of autocorrelation processes in queues (Balcioglu et al., 2008). Some works have shown examples of autocorrelation in data from industrial plants (Luxhoj and Shyur, 1995; Melamed and Hill, 1995; Mertens et al. 2009; Schomig and Mittler, 1995; Young and Winistorfer, 2001).

However, Hejn Nielsen (2007) casts doubts on the practical relevance of some of the autocorrelation models employed by the authors previously mentioned. His results also provide evidence that autocorrelation, if present, has a relevant impact in process performance. But circumstances present in manufacturing plants may reduce its importance; for example, due to the adoption of certain process control rules. Also, the effects might either be favorable or unfavorable depending on the autocorrelation pattern. If autocorrelation is included in a model, great care should be taken when choosing the model parameters. High order terms might be necessary in some situations.

The section 2 of this paper presents a simulation experiment conducted in order to illustrate the different effects on process performance that can be related to autocorrelation in cycle times. Autocorrelation is introduced by means of autoregressive processes of up to order five. This form of autocorrelation is introduced at a process cycle execution time scale and is similar to the models considered by previous authors.

However, autocorrelation in a manufacturing process may not only be introduced at a cycle execution time scale. Sources of variation affecting performance at different time scales may also display autocorrelation. For instance, let's consider a hypothetical case of daily variations in performance related to human factors in a manufacturing system. External factors to the process, such as physiological conditions, motivation or others may affect the labor's performance and vary from one day to the next (Baines et al. 2004). Furthermore, these variations in performance might display autocorrelation due to persistent factors that affect performance in successive days. Similar examples might be constructed referring to variations in demand (whose time series ought to display autocorrelation) and other external factors such as weather or the social environment.

Let's consider the case of variability in task times happening at two time scales: task execution and time periods of length $T$ (these time periods might be hours, days, months, etc.). Then, a general model for the task duration would be as given by (2).

$$
t_{i, c} \sim G_{c}\left(\mu_{i}\right)
$$

where $t_{i}$ is the random variable that represents the duration of the $c^{\text {th }}$ task execution during the time period $i, \mu_{i}$ is the average task duration during the time period $i$ and $G_{c}$ is a general distribution that might depend on other parameters as well as $\mu_{i}$. Variability at the time scale given by periods of length $T$ can be described by the general distribution of task duration averages:

$$
\mu_{i} \sim G_{d}\left(\mu, \mu_{i-1}, \mu_{i-2}, \ldots\right)
$$

where $\mu$ is the long term average task duration, $G_{d}$ a general distribution that might depend on other parameters as well as $\mu$ and the previous observations (case of autocorrelation). Figure 1 shows an example of such a process in which $T=5$.

Section 3 presents a series of simulation experiments conducted on three general process arrangements (serial, assembly and disassembly lines) in which autoregressive processes are employed for mod- 


\section{Crespo Pereira, D. del Rio Vilas, N. Rego Monteil, R. Rios Prado and A. Garcia del Valle}

eling variations in process parameters arising at a time scale different from the corresponding to process cycle execution.

Such a type of variation was found to have practical relevance in the analysis of a natural slate tiles manufacturing process (Crespo Pereira et al, 2012). In this actual case, the variability in the properties of the input materials caused several performance parameters to change, affecting both flow balance and the overall plant performance. The time series displayed significant levels of autocorrelation. Section 4 revisits this case presenting a new time series analysis of performance metrics. The paper ends with some conclusions on the modeling of autocorrelation in manufacturing.

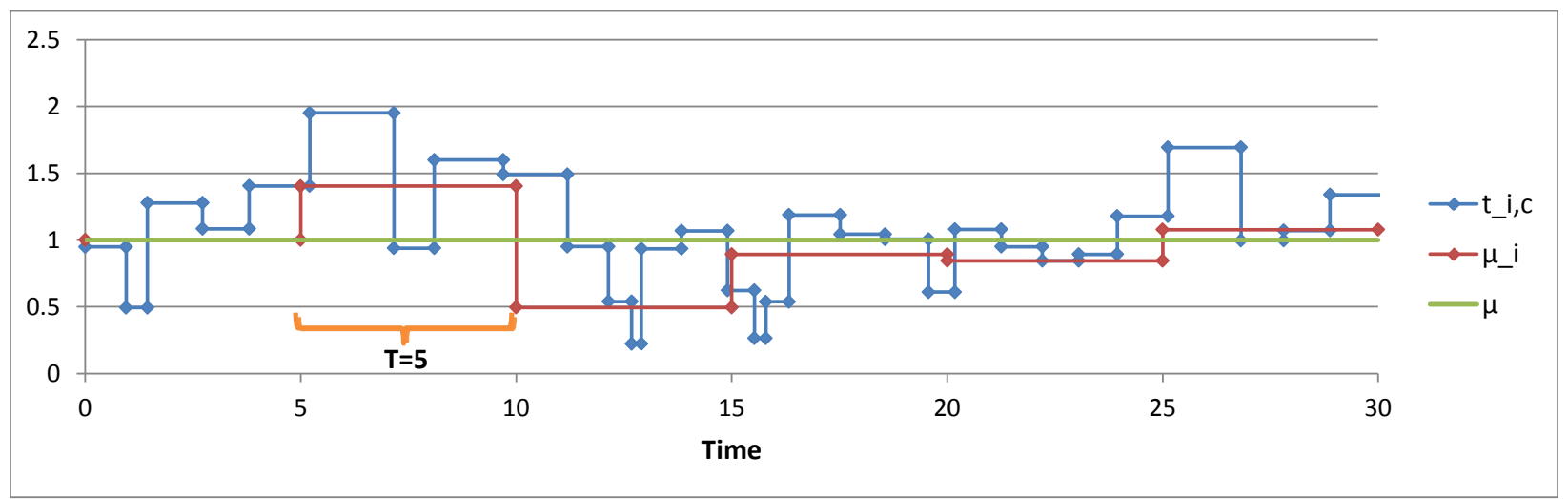

Figure 1. Example of a task duration time series with variability at two time scales: task execution and periods of length 5 .

\section{AUTOCORRELATION IN CYCLE TIMES}

The following simulation experiment was conducted in order to illustrate the complexity in process behavior caused by autocorrelation. A serial line consisting of ten machines with intermediate limited capacity buffers was simulated in Quest V5R20 (Figure 2). Machines cycle times were modeled as an autoregressive process of order up to five. Coefficients were arranged so that the coefficient of variation was equal to $1 / 3$ for all the cases. This value is representative of variability in human tasks duration.

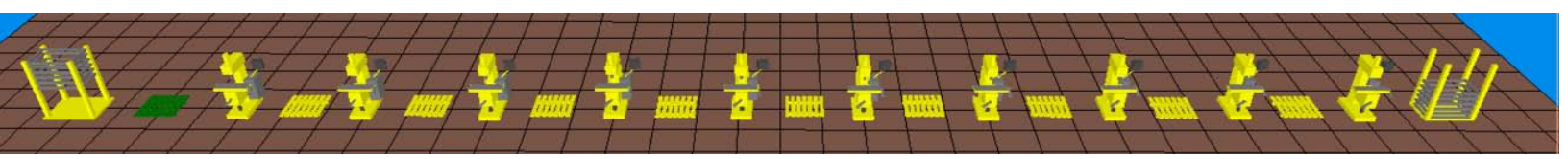

Figure 2. Quest model of a 10 machines serial line.

Then, the cycle time at the $c^{\text {th }}$ execution of the process of a machine is given by (4):

$t_{c}=\mu+\varphi_{1} \cdot\left(t_{c-1}-\mu\right)+\varphi_{2} \cdot\left(t_{c-2}-\mu\right)+\varphi_{3} \cdot\left(t_{c-3}-\mu\right)+\varphi_{4} \cdot\left(t_{c-4}-\mu\right)+\varphi_{5} \cdot\left(t_{c-5}-\mu\right)+\varepsilon_{c}$

Where $\mu=10$ is the cycle time average, $\varphi_{k}, k=1, \ldots, 5$ are the coefficients of the AR5 model and $\varepsilon_{c} \sim N\left(0, \sigma_{\delta}\right)$ is a white noise process following a normal distribution with $\sigma_{\delta}$ deviation.

Table 1 shows eight combinations of parameters values corresponding to eight scenarios that were studied. In addition, $\sigma_{\delta}$ has been adjusted for each scenario so that $\frac{\sqrt{\operatorname{Var}\left(t_{c}\right)}}{\mu}=1 / 3$. Thus, only the autocorrelation pattern is altered but the coefficient of variation is kept constant. Scenario O represents the case of no autocorrelation. Scenario A contains only positive first order autocorrelation. Scenarios B and B' represent cases with higher order autocorrelation, positive in one case and negative in the other. Sce- 
narios $\mathrm{C}$ and $\mathrm{C}^{\prime}$ represent cases in which there is jointly a low order autocorrelation effect and a higher $\operatorname{order}\left(5^{\text {th }}\right.$ order$)$ opposite effect. D and $D^{\prime}$ are variations on the previous scenario.

Table 1. Scenarios for the simulation experiment.

\begin{tabular}{|l|c|c|c|c|c|c|}
\hline Scenario & $\varphi_{1}$ & $\varphi_{2}$ & $\varphi_{3}$ & $\varphi_{4}$ & $\varphi_{5}$ & $\sigma_{\delta}$ \\
\hline O & 0.00 & 0.00 & 0.00 & 0.00 & 0.00 & 3.333 \\
\hline A & 0.50 & 0.00 & 0.00 & 0.00 & 0.00 & 2.350 \\
\hline B & 0.20 & 0.20 & 0.20 & 0.00 & 0.00 & 2.892 \\
\hline B $^{\prime}$ & -0.20 & -0.20 & -0.20 & 0.00 & 0.00 & 3.019 \\
\hline C & 0.50 & 0.00 & 0.00 & 0.00 & -0.50 & 3.207 \\
\hline C' $^{\prime}$ & -0.50 & 0.00 & 0.00 & 0.00 & 0.50 & 2.578 \\
\hline D & 0.30 & 0.20 & 0.00 & -0.20 & -0.30 & 2.984 \\
\hline D' & -0.30 & -0.20 & 0.00 & 0.20 & 0.30 & 1.995 \\
\hline
\end{tabular}

If times are constant, the capacity of this serial line would be 0.1 products per time unit. However, due to the limited capacity of intermediate buffers along with variability, this throughput rate is not achieved. Interarrival times were introduced following a normal distribution $N(10,1)$. The line performance, defined as the percentage of the maximum capacity, was evaluated for each scenario by means of 30 simulation runs. Table 2 shows the obtained results for buffer capacity equal to 1, 3 and 5 . Average line performance $(\bar{\eta})$, its standard deviation $\left(s_{\eta}\right)$ and the $95 \%$ confidence interval for the mean $\left(C I_{\eta}\right)$ are given for each buffer capacity. Figure 3 presents the same results in a chart.

Table 2. Scenarios for the simulation experiment.

\begin{tabular}{|c|c|c|c|c|c|c|c|c|c|}
\hline \multirow{2}{*}{ Scenario } & \multicolumn{4}{|c|}{ Buffer capacity 1} & \multicolumn{3}{c|}{ Buffer capacity 3 } & \multicolumn{3}{c|}{ Buffer capacity 5 } \\
\cline { 2 - 10 } & $\overline{\boldsymbol{\eta}}$ & $\boldsymbol{s}_{\boldsymbol{\eta}}$ & $\boldsymbol{C I}_{\boldsymbol{\eta}}$ & $\overline{\boldsymbol{\eta}}$ & $\boldsymbol{s}_{\boldsymbol{\eta}}$ & $\boldsymbol{C I}_{\boldsymbol{\eta}}$ & $\overline{\boldsymbol{\eta}}$ & $\boldsymbol{s}_{\boldsymbol{\eta}}$ & $\boldsymbol{C I}_{\boldsymbol{\eta}}$ \\
\hline $\mathrm{O}$ & $86.43 \%$ & $0.94 \%$ & $0.35 \%$ & $93.38 \%$ & $1.39 \%$ & $0.52 \%$ & $94.87 \%$ & $1.26 \%$ & $0.47 \%$ \\
\hline $\mathrm{A}$ & $81.74 \%$ & $0.97 \%$ & $0.35 \%$ & $88.47 \%$ & $1.30 \%$ & $0.46 \%$ & $91.47 \%$ & $1.32 \%$ & $0.47 \%$ \\
\hline $\mathrm{B}$ & $81.42 \%$ & $0.86 \%$ & $0.31 \%$ & $86.57 \%$ & $1.58 \%$ & $0.57 \%$ & $89.55 \%$ & $1.17 \%$ & $0.42 \%$ \\
\hline $\mathrm{B}$ & $92.14 \%$ & $0.39 \%$ & $0.14 \%$ & $97.41 \%$ & $0.69 \%$ & $0.25 \%$ & $98.23 \%$ & $0.72 \%$ & $0.26 \%$ \\
\hline $\mathrm{C}$ & $76.55 \%$ & $0.84 \%$ & $0.30 \%$ & $88.31 \%$ & $1.07 \%$ & $0.38 \%$ & $93.37 \%$ & $0.95 \%$ & $0.34 \%$ \\
\hline $\mathrm{C}$ & $91.49 \%$ & $0.51 \%$ & $0.18 \%$ & $95.78 \%$ & $0.66 \%$ & $0.24 \%$ & $96.80 \%$ & $0.84 \%$ & $0.30 \%$ \\
\hline $\mathrm{D}$ & $80.67 \%$ & $0.86 \%$ & $0.31 \%$ & $91.83 \%$ & $0.80 \%$ & $0.29 \%$ & $95.58 \%$ & $1.10 \%$ & $0.39 \%$ \\
\hline $\mathrm{D}$ & $95.04 \%$ & $0.36 \%$ & $0.13 \%$ & $97.78 \%$ & $0.63 \%$ & $0.22 \%$ & $98.10 \%$ & $0.62 \%$ & $0.22 \%$ \\
\hline
\end{tabular}

As it can be seen in Table 2 and Figure 3, there is an evident impact of autocorrelation in line performance which is indeed affected by buffer capacity. Positive autocorrelation scenarios (A and B) deteriorate performance when compared to the base scenario with independence in cycle times $(\mathrm{O})$, irrespective of buffer size. Scenarios with negative first order autocorrelations (B', C' and D') demonstrate an enhanced performance. Scenarios C and D (low order positive autocorrelation, high order negative autocorrelation) show a more complex effect on performance depending on buffer capacity. For small buffer capacity, equal to 1, performance is as low or even lower than scenarios A and B. But once buffer capacity is increased, performance achieves similar levels to the base scenario or even higher. These results support evidence from previous research regarding the potential positive or negative effects driven by autocorrelation on process performance (Hejn Nielsen, 2007). 


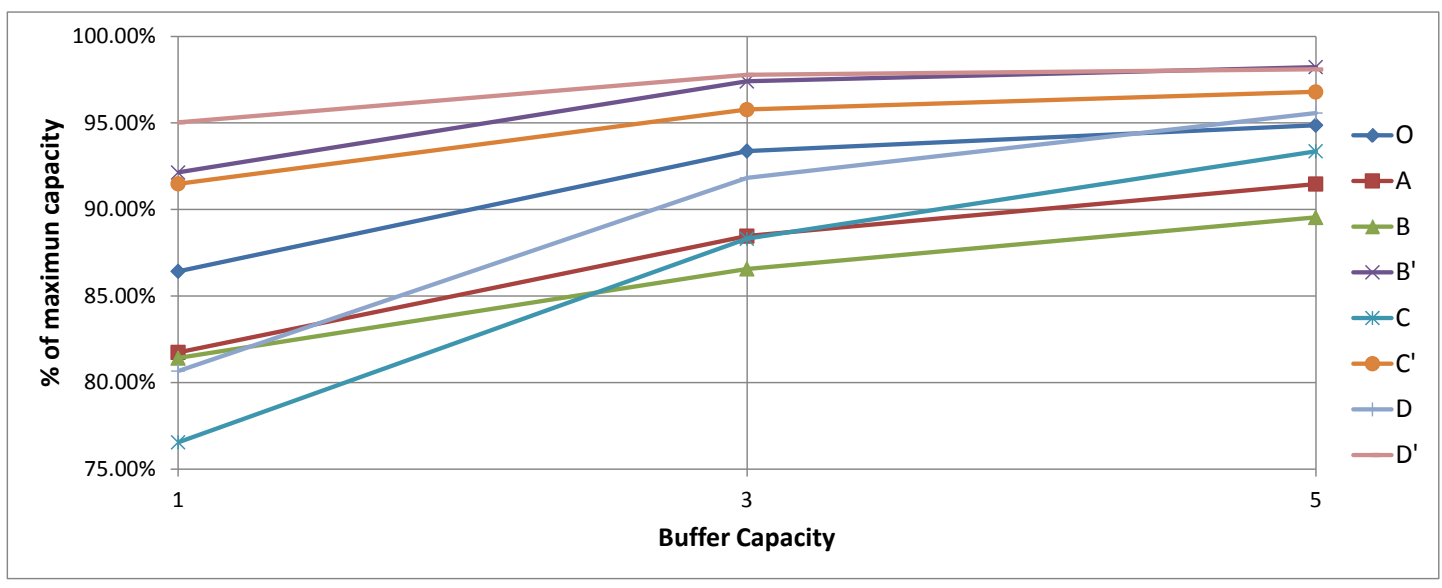

Figure 3. Line performance for different buffer capacities.

\section{AUTOCORRELATION AT DIFFERENT TIME SCALES}

In this section, the impact in performance from average cycle times following first order autoregressive processes is studied for three general types of manufacturing processes:

- A serial line with three machines

- An assembly process in which the product flow from two parallel machines converges in a single workstation.

- A disassembly process in which, after an initial processing in one machine, the product flow is randomly divided in two parallel lines with one machine each.

All the process parameters have been chosen similarly so that the results from the three models may be compared more easily. Accordingly:

- Input parts to the system are always available in the sources.

- Machines cycle times are calculated as $t_{i, c}=\mu_{i} \cdot \varepsilon_{i, c}$ where $t_{i, c}$ is the duration of cycle $c$ in the time period $i, \mu_{i}$ is the cycle time average in the period $i$ and $\varepsilon_{i, c}$ follows a lognormal distribution with average 1 and standard deviation $\sigma_{c}=1 / 3$.

- Intermediate buffers capacities are set to one in all the experiments conducted (as a means for strengthening losses of performance due to blocking and starvation).

- The response of the simulation model is the line performance $\eta$ calculated as the percentage of the maximum throughput (achievable under non-variability conditions with the long term cycle time average $\mu=1$ and equal to 1 product per time unit) that is observed in the model.

For the serial line and the assembly line, every $T$ time units the average cycle time $\mu_{i}$ is generated following a first order autoregressive model (AR1) as shown in (5).

$$
\mu_{i}=\mu+\beta \cdot\left(\mu_{i-1}-\mu\right)+\delta_{i}
$$

where $\mu=1$ is the long term average cycle time and $\delta_{i}$ is a white noise process with standard deviation $\sigma_{\delta}$.

\subsection{Serial Line}

The serial line modeled in Quest is shown in Figure 4. The model contains a source, three machines, two capacitated buffers, a sink and two controllers that generate the time series and sample data at fixed periods of time corresponding to the time series step $T$.

The experimentation consisted of 200 simulation runs of 2000 time units (with a warm up time of 100 units in which statistics were not collected) for each combination of the following parameters: $T=$ $\{5,10,50,100\}, \beta=\{-0.8,-0.4,0.0,0.4,0.8\}$ and $\sigma_{\delta}=\{0,0.1\}$. Table 3 summarizes the results. As it can 


\section{Crespo Pereira, D. del Rio Vilas, N. Rego Monteil, R. Rios Prado and A. Garcia del Valle}

be seen, autocorrelation has a negative impact in performance for both positive and negative autocorrelation coefficients. The impact is greater when $T$ is increased.

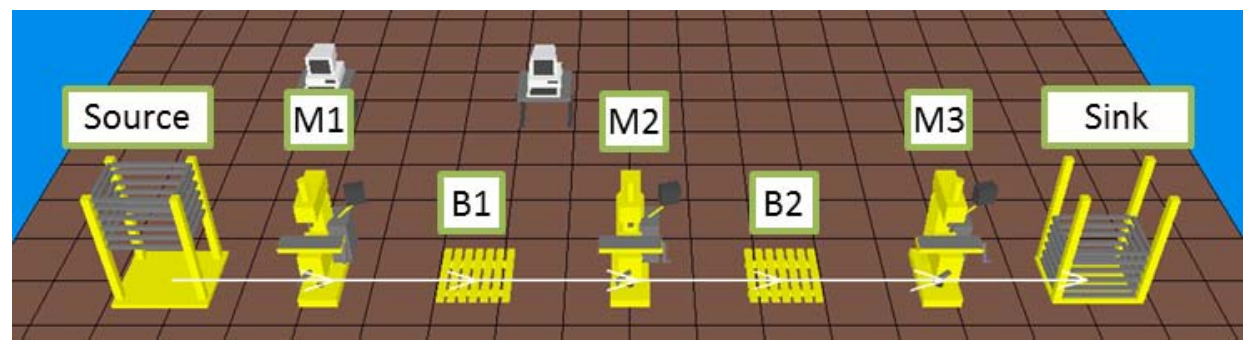

Figure 4. Serial line model.

Table 3. Line performance $\eta$ (95\% confidence level).

\begin{tabular}{|c|c|c|c|c|c|c|}
\hline \multirow{2}{*}{$\boldsymbol{T}$} & $\boldsymbol{\sigma}_{\boldsymbol{\delta}}=\mathbf{0}$ & \multicolumn{5}{|c|}{$\boldsymbol{\sigma}_{\boldsymbol{\delta}}=\mathbf{0 . 1}$} \\
\cline { 2 - 7 } & $\boldsymbol{\beta}=\mathbf{0 . 0}$ & $\boldsymbol{\beta}=-\mathbf{0 . 8}$ & $\boldsymbol{\beta}=-\mathbf{0 . 4}$ & $\boldsymbol{\beta}=\mathbf{0 . 0}$ & $\boldsymbol{\beta}=\mathbf{0 . 4}$ & $\boldsymbol{\beta}=\mathbf{0 . 8}$ \\
\hline $\mathbf{5}$ & $92.21 \% \pm 0.07 \%$ & $90.88 \% \pm 0.08 \%$ & $91.20 \% \pm 0.08 \%$ & $90.92 \% \pm 0.08 \%$ & $90.29 \% \pm 0.09 \%$ & $87.28 \% \pm 0.19 \%$ \\
\hline $\mathbf{1 0}$ & $92.18 \% \pm 0.07 \%$ & $89.22 \% \pm 0.10 \%$ & $90.53 \% \pm 0.08 \%$ & $90.41 \% \pm 0.10 \%$ & $89.78 \% \pm 0.11 \%$ & $87.13 \% \pm 0.26 \%$ \\
\hline $\mathbf{5 0}$ & $92.17 \% \pm 0.07 \%$ & $87.39 \% \pm 0.20 \%$ & $89.60 \% \pm 0.12 \%$ & $89.69 \% \pm 0.14 \%$ & $89.46 \% \pm 0.21 \%$ & $87.25 \% \pm 0.55 \%$ \\
\hline $\mathbf{1 0 0}$ & $92.20 \% \pm 0.07 \%$ & $87.41 \% \pm 0.29 \%$ & $89.34 \% \pm 0.16 \%$ & $89.72 \% \pm 0.18 \%$ & $89.24 \% \pm 0.29 \%$ & $86.87 \% \pm 0.64 \%$ \\
\hline
\end{tabular}

\subsection{Assembly Line}

The assembly line is based on the previous case. Two sources in parallel create parts which are processed in machines 1 and 2 (Figure 5). They are later assembled in the machine 3 requiring one part from each source to be batched together. 200 simulation runs of 2000 time units (with a warm up time of 100 units in which statistics were not collected) were performed for each combination of the following parameters: $T=\{5,10,50,100\}, \beta=\{-0.8,-0.4,0.0,0.4,0.8\}$ and $\sigma_{\delta}=\{0,0.1\}$. This assembly line shows the same behavior as the serial line (Table 4).

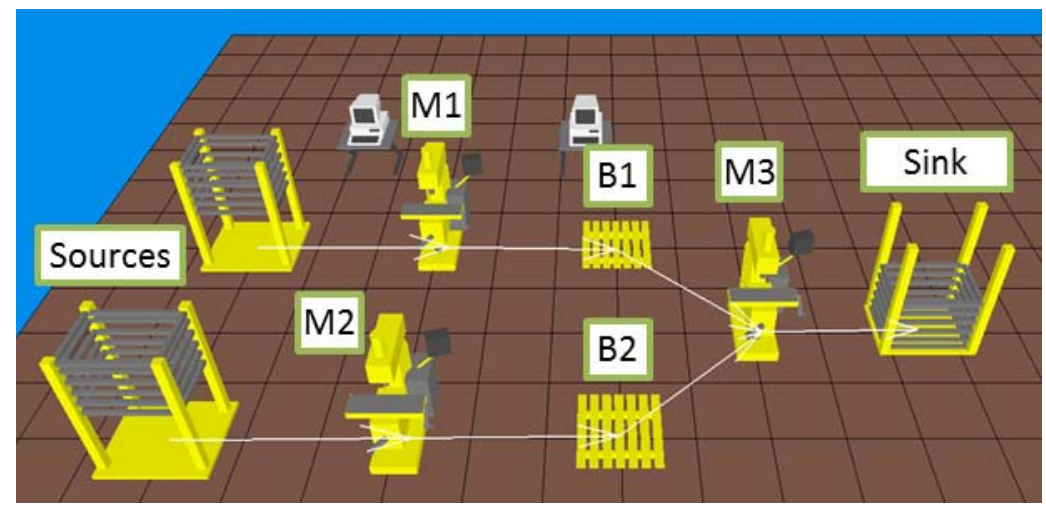

Figure 5. Assembly line model.

Table 4. Line performance $\eta$ for different model parameters (95\% confidence level).

\begin{tabular}{|c|c|c|c|c|c|c|}
\hline \multirow{2}{*}{$\boldsymbol{T}$} & $\boldsymbol{\sigma}_{\boldsymbol{\delta}}=\mathbf{0}$ & \multicolumn{5}{|c|}{$\boldsymbol{\sigma}_{\boldsymbol{\delta}}=\mathbf{0 . 1}$} \\
\cline { 2 - 7 } & $\boldsymbol{\beta}=\mathbf{0 . 0}$ & $\boldsymbol{\beta}=-\mathbf{0 . 8}$ & $\boldsymbol{\beta}=-\mathbf{0 . 4}$ & $\boldsymbol{\beta}=\mathbf{0 . 0}$ & $\boldsymbol{\beta}=\mathbf{0 . 4}$ & $\boldsymbol{\beta}=\mathbf{0 . 8}$ \\
\hline $\mathbf{5}$ & $92.26 \% \pm 0.07 \%$ & $90.96 \% \pm 0.08 \%$ & $91.39 \% \pm 0.07 \%$ & $91.15 \% \pm 0.09 \%$ & $90.40 \% \pm 0.10 \%$ & $87.39 \% \pm 0.19 \%$ \\
\hline $\mathbf{1 0}$ & $92.24 \% \pm 0.06 \%$ & $89.13 \% \pm 0.10 \%$ & $90.62 \% \pm 0.09 \%$ & $90.47 \% \pm 0.09 \%$ & $89.87 \% \pm 0.11 \%$ & $87.32 \% \pm 0.31 \%$ \\
\hline $\mathbf{5 0}$ & $92.18 \% \pm 0.06 \%$ & $87.16 \% \pm 0.22 \%$ & $89.54 \% \pm 0.13 \%$ & $89.79 \% \pm 0.13 \%$ & $88.93 \% \pm 0.14 \%$ & $86.30 \% \pm 0.49 \%$ \\
\hline $\mathbf{1 0 0}$ & $92.19 \% \pm 0.06 \%$ & $87.20 \% \pm 0.32 \%$ & $89.29 \% \pm 0.19 \%$ & $89.87 \% \pm 0.19 \%$ & $89.09 \% \pm 0.27 \%$ & $87.14 \% \pm 0.80 \%$ \\
\hline
\end{tabular}




\subsection{Disassembly Line}

For the disassembly line, autocorrelation was introduced in the definition of fractions of disassembled products which are processed in each parallel line instead of in cycle times averages. The model shown in Figure 6 consists of a first machine that performs the disassembling operation. For each input part two new parts are created. These two parts are randomly assigned to each one of the following parallel lines with probability $p_{i}$ where $i$ is the index of the time period of length $T$ as previously defined. Thus, parts are routed to machine 2 with probability $p_{i}$ and to machine 3 with probability $\left(1-p_{i}\right)$. The variability in $p_{i}$ is modeled by an AR1 process as given in (6) in which $p=0.5$ is the long term average and $\xi_{i}$ is a white noise process with standard deviation $\sigma_{p}$. As shown in Table 5, no relevant effects due to autocorrelation were found.

$$
p_{i}=p+\beta_{p} \cdot\left(p_{i-1}-p\right)+\xi_{i}
$$

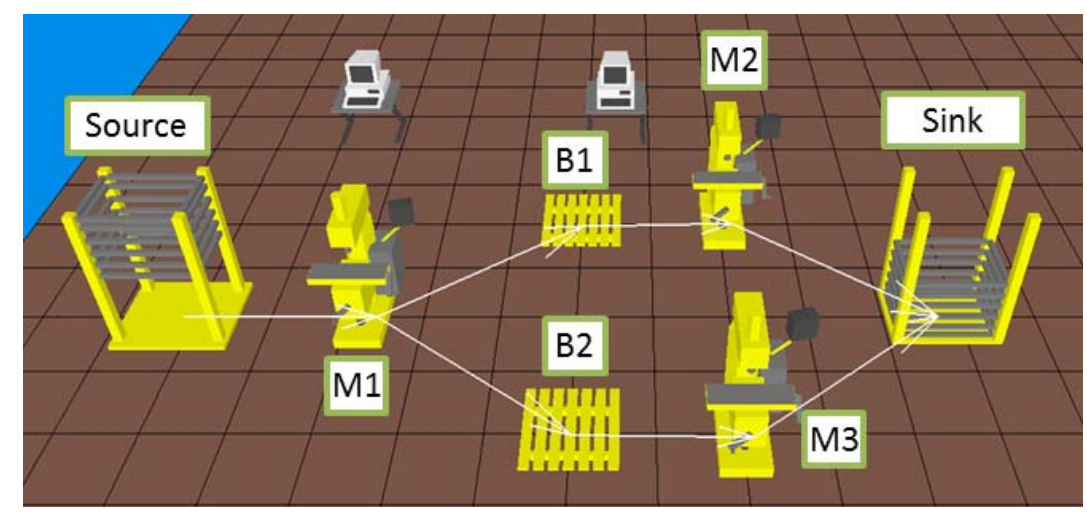

Figure 6. Disassembly line model.

Table 5. Average buffer content for different model parameters ( $95 \%$ confidence level).

\begin{tabular}{|c|c|c|c|c|c|c|}
\hline \multirow{2}{*}{$\boldsymbol{T}$} & $\boldsymbol{\sigma}_{\boldsymbol{\delta}}=\mathbf{0}$ & \multicolumn{5}{|c|}{$\boldsymbol{\sigma}_{\boldsymbol{\delta}}=\mathbf{0 . 1}$} \\
\cline { 2 - 7 } & $\boldsymbol{\beta}=\mathbf{0 . 0}$ & $\boldsymbol{\beta}=-\mathbf{0 . 8}$ & $\boldsymbol{\beta}=-\mathbf{0 . 4}$ & $\boldsymbol{\beta}=\mathbf{0 . 0}$ & $\boldsymbol{\beta}=\mathbf{0 . 4}$ & $\boldsymbol{\beta}=\mathbf{0 . 8}$ \\
\hline $\mathbf{5}$ & $75.06 \% \pm 0.10 \%$ & $75.06 \% \pm 0.10 \%$ & $75.14 \% \pm 0.09 \%$ & $75.11 \% \pm 0.08 \%$ & $75.04 \% \pm 0.09 \%$ & $75.06 \% \pm 0.08 \%$ \\
\hline $\mathbf{1 0}$ & $75.04 \% \pm 0.11 \%$ & $74.95 \% \pm 0.08 \%$ & $74.96 \% \pm 0.09 \%$ & $75.11 \% \pm 0.08 \%$ & $75.02 \% \pm 0.10 \%$ & $75.03 \% \pm 0.10 \%$ \\
\hline $\mathbf{5 0}$ & $74.93 \% \pm 0.10 \%$ & $75.00 \% \pm 0.08 \%$ & $75.04 \% \pm 0.10 \%$ & $74.99 \% \pm 0.09 \%$ & $75.02 \% \pm 0.10 \%$ & $74.98 \% \pm 0.07 \%$ \\
\hline $\mathbf{1 0 0}$ & $74.94 \% \pm 0.11 \%$ & $75.03 \% \pm 0.08 \%$ & $75.19 \% \pm 0.09 \%$ & $74.93 \% \pm 0.09 \%$ & $74.97 \% \pm 0.09 \%$ & $74.99 \% \pm 0.09 \%$ \\
\hline
\end{tabular}

\section{CASE STUDY}

\subsection{Process Description}

A manufacturing plant of natural slate tiles was analyzed by means of discrete events simulation within the frame of a co-operation R\&D project (Rego Monteil et al. 2010; del Rio Vilas et al. 2009). Since this is a labor-intensive process that deals with natural products, it is a paradigmatic case of highly variable manufacturing environment (Crespo Pereira et al. 2012).

The production process begins with the extraction of irregular pieces of slate from a quarry, which are then transported to the manufacturing plant. There, they are first sawed into blocks and then manually split into pieces of a standard thickness. Afterwards, the resulting piles of plates are cut into different shapes and sizes according to the commercial formats. Finally, tiles are classified by standardized grades of quality and then packed in crate pallets. Three main formats are produced $(32 \times 22 \mathrm{~mm}, 30 \times 20 \mathrm{~mm}$ and $27 \times 18 \mathrm{~mm})$ as well as three quality grades (first, traditional and standard) and two thickness ( $3.5 \mathrm{~mm}$ and $4.5 \mathrm{~mm})$. 


\section{Crespo Pereira, D. del Rio Vilas, N. Rego Monteil, R. Rios Prado and A. Garcia del Valle}

The process was initially out of statistical control and neither task duration standards nor systematic production control rules were available. Decisions were taken in a reactive mode according to intuitive rules developed by the plant foreman. As a means for improving the knowledge on the process behavior, data were collected from production records, video recording and interviews with workers. A simulation model was developed and implemented in Quest V5R20 aiding in the process analysis stage by indicating information requirements and the relations between elements.

The first developed simulation model (M1) (in which no autocorrelation effects were considered) showed some problems in the validation step. Relevant differences in production variability and work in process levels were found. After a deeper analysis of the input data time series, a second model (M2) was developed in which daily variations in process parameters were modeled by means of autoregressive processes. The second model presented a closer behavior to the real system so it was employed in later steps. A new proposed layout was analyzed by means of M2 (Crespo Pereira et al. 2012), showing a reduction in non-value added activities along with an increase in plant capacity. Figure 7 presents the simulation model for the original and proposed layouts.

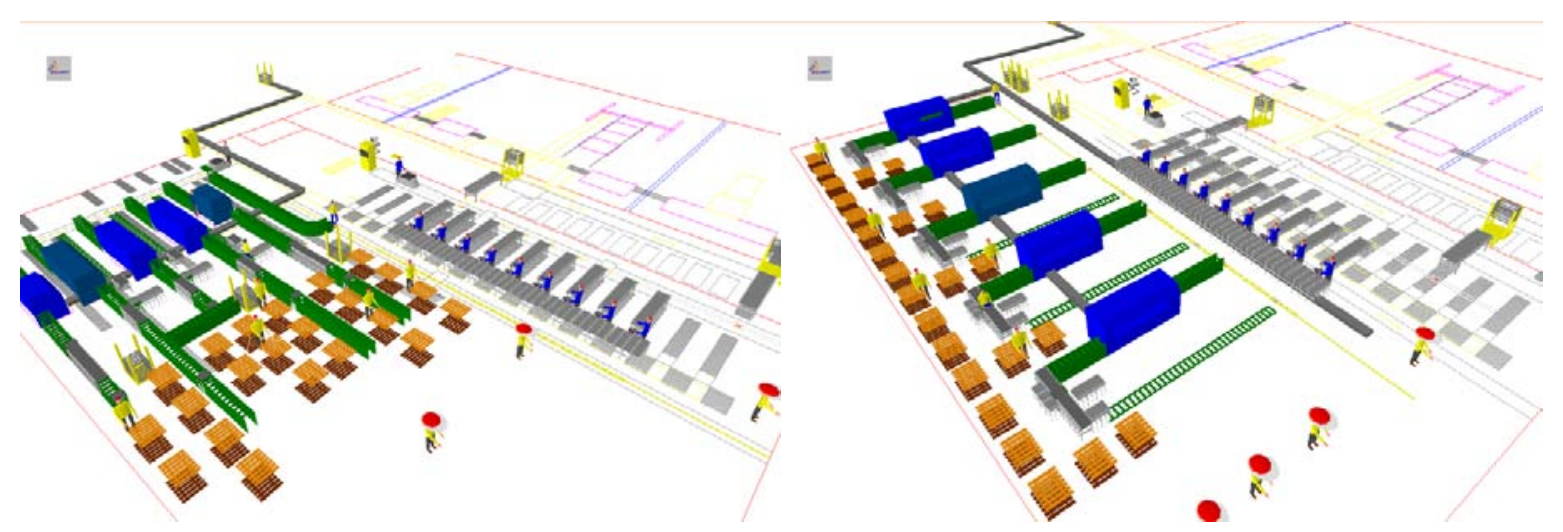

Figure 7. Quest models for the actual layout (left) and new proposed layout (right).

\subsection{Autocorrelation Effects}

In this system, the following seven key parameters were considered due to their impact in performance:

- $\tau_{S U}$ : Utilization rate of split blocks. It represents the percentage of the blocks material that is transformed into tiles.

- $\tau_{T F}$ : Rate of target format slates produced in the cutting operation. The target format is usually $32 \times 22 \mathrm{~mm}$. A tile cut in $32 \times 22 \mathrm{~mm}$ size may be recirculated in the classification step to be cut in a smaller size.

- $\tau_{r e j}$ : Rejection rate in the classification step. Rejected tiles are wasted.

- $\tau_{32}$ : Rate of 32 format slates finally produced in the factory.

- $\tau_{F}$ : Rate of First grade slates produced in the factory.

- $\tau_{\text {recirc }}$ : Rate of slates recirculated after the classification step for cutting into smaller sizes.

- $\tau_{\text {thick }}$ : Rate of slates classified as of $4.5 \mathrm{~mm}$ thickness.

Since the input rate of blocks into the factory is kept constant, the aforementioned parameters determine the throughput rate for the different grades and formats commercialized. Thus, they affect both production costs and revenues. These parameters vary along time depending on the extracted material properties (which are heterogeneous throughout the quarry). Daily production records were used to estimate their values.

In the first developed model (M1), these parameters were introduced by their mean values. Once results from the model were found to underestimate variability, their time series were analyzed. First of all, the time series were normalized and a Principal Components Analysis (PCA) was performed aiming at avoiding problems of collinearity. Four principal components were found to account for the $80.30 \%$ of 


\section{Crespo Pereira, D. del Rio Vilas, N. Rego Monteil, R. Rios Prado and A. Garcia del Valle}

the variance. Their values are shown in Table 6. Then, first order autoregressive models (as given in (7)) were fitted for these four components. Terms of higher autocorrelation order were rejected due to low significance. Table 7 shows the fitted models (indicated in the "System" rows).

$$
c_{i, t}=\beta_{1, i} \cdot c_{i, t-1}+\xi_{i, t}
$$

where $c_{i, t}$ is the value of the $i^{\text {th }}$ principal component in day $t, \beta_{1, i}$ the first order autocorrelation coefficient and $\xi_{i, t} \sim N\left(0, \sigma_{c, i}\right)$ is a white noise process of standard deviation $\sigma_{c, i}$.

The fitted time series were implemented in the Quest simulation model. Thus, the two modeling approaches could be compared. In M1, process parameters average values were kept constant along the simulation and variability was only introduced in cycle time distributions. In M2, daily average values of the process parameters were generated following the fitted AR models and variability was also introduced in cycle time distributions. More details on the models implementation are provided by Crespo Pereira et al. (2012)

Table 6. Principal Components Analysis of the parameters time series.

\begin{tabular}{|c|c|c|c|c|}
\hline \multirow{2}{*}{ Parameter } & \multicolumn{4}{|c|}{ Principal Components } \\
\cline { 2 - 5 } & $\boldsymbol{c}_{\mathbf{1}}$ & $\boldsymbol{c}_{\mathbf{2}}$ & $\boldsymbol{c}_{\mathbf{3}}$ & $\boldsymbol{c}_{\mathbf{4}}$ \\
\hline$\tau_{S U}$ & 0.466 & -0.254 & - & -0.152 \\
\hline$\tau_{T F}$ & - & 0.566 & -0.377 & 0.236 \\
\hline $1-\tau_{r e j}$ & -0.537 & 0.162 & 0.116 & - \\
\hline$\tau_{32}$ & 0.451 & 0.232 & 0.109 & 0.151 \\
\hline$\tau_{F}$ & - & -0.343 & -0.819 & -0.258 \\
\hline$\tau_{\text {recirc }}$ & -0.492 & - & -0.234 & - \\
\hline$\tau_{\text {thick }}$ & - & -0.397 & -0.112 & 0.897 \\
\hline
\end{tabular}

In order to validate models M1 and M2, production records similar to those available from the factory were generated by means of simulation. The time series analysis conducted on real data was applied to the simulated data. Table 7 shows the variances and the autocorrelations of the principal components time series fitted for the real data and for the generated data from both simulation models. M2 provides a more precise approximation to real data than M1. Standard deviations and first order autocorrelation coefficients are closer to real values for M2 than those from M1. The Table also shows the p-value of the t-test performed for testing the null hypothesis $\beta_{1, i}=0$.

Other process variables were also considered in the validation stage. Four relevant variables are the content of buffers previous to cutting and classification operations and the utilization of splitters and packers. The autocorrelation function of daily averages time series were compared for M1 and M2. Real values from data were not available in this case, but M2 was considered to be a more realistic representation of the system because of the previous results. Figure 8 shows the autocorrelation function generated by M1 and M2 for these variables. It represents the autocorrelation coefficients $\rho_{l}$ as a function of the lag $l$ as defined by (1). As it can be clearly noticed, models M1 and M2 display quite different autocorrelation functions. This result shows that the behavior of process variables may be severely affected by the consideration of autocorrelation effects in some process variables.

Finally, Figure 9 depicts the simulated time series of daily average connection buffer contents for both models. M2 shows a more irregular pattern of buffer contents that better corresponds with descriptions of the system behavior provided by the plant managers. Thus, this result supports the previous statement that M2 is a better representation of the plant. To conclude with, it can be said that in this case the i.i.d. assumption of process parameters would lead to an underestimation of both process variability and buffer contents. 
D. Crespo Pereira, D. del Rio Vilas, N. Rego Monteil, R. Rios Prado and A. Garcia del Valle

Table 7. Comparison between models generated results and real data.

\begin{tabular}{|c|c|c|c|c|c|}
\hline & & $\mathrm{c}_{1}$ & $\mathrm{c}_{2}$ & $\mathrm{C}_{3}$ & $\mathrm{c}_{4}$ \\
\hline Std. Deviation & System & 1.667 & 1.344 & 0.981 & 0.914 \\
\hline \multirow{2}{*}{$\left(\sqrt{\operatorname{var}\left(c_{i, t}\right)}\right)$} & Model 1 & 1.465 & 1.158 & 1.073 & 1.010 \\
\hline & Model 2 & 1.571 & 1.363 & 0.988 & 0.963 \\
\hline \multirow{3}{*}{$\begin{array}{l}\text { AR1 coef. } \\
\qquad\left(\boldsymbol{\beta}_{1, i}\right)\end{array}$} & System & 0.714 & 0.619 & 0.451 & 0.211 \\
\hline & Model 1 & -0.539 & -0.474 & -0.413 & 0.014 \\
\hline & Model 2 & 0.432 & 0.285 & 0.354 & 0.153 \\
\hline \multirow{3}{*}{$\begin{array}{c}\beta_{1, i} \text { t-test } \\
\text { p-value }\end{array}$} & System & $<2 \mathrm{e}-16$ & $<2 \mathrm{e}-16$ & $1.17 \mathrm{e}-10$ & 0.00493 \\
\hline & Model 1 & $<2 \mathrm{e}-16$ & $2.44 \mathrm{e}-14$ & $1.34 \mathrm{e}-10$ & 0.844 \\
\hline & Model 2 & $1.88 \mathrm{e}-09$ & 0.000162 & 0.00000194 & 0.049 \\
\hline
\end{tabular}
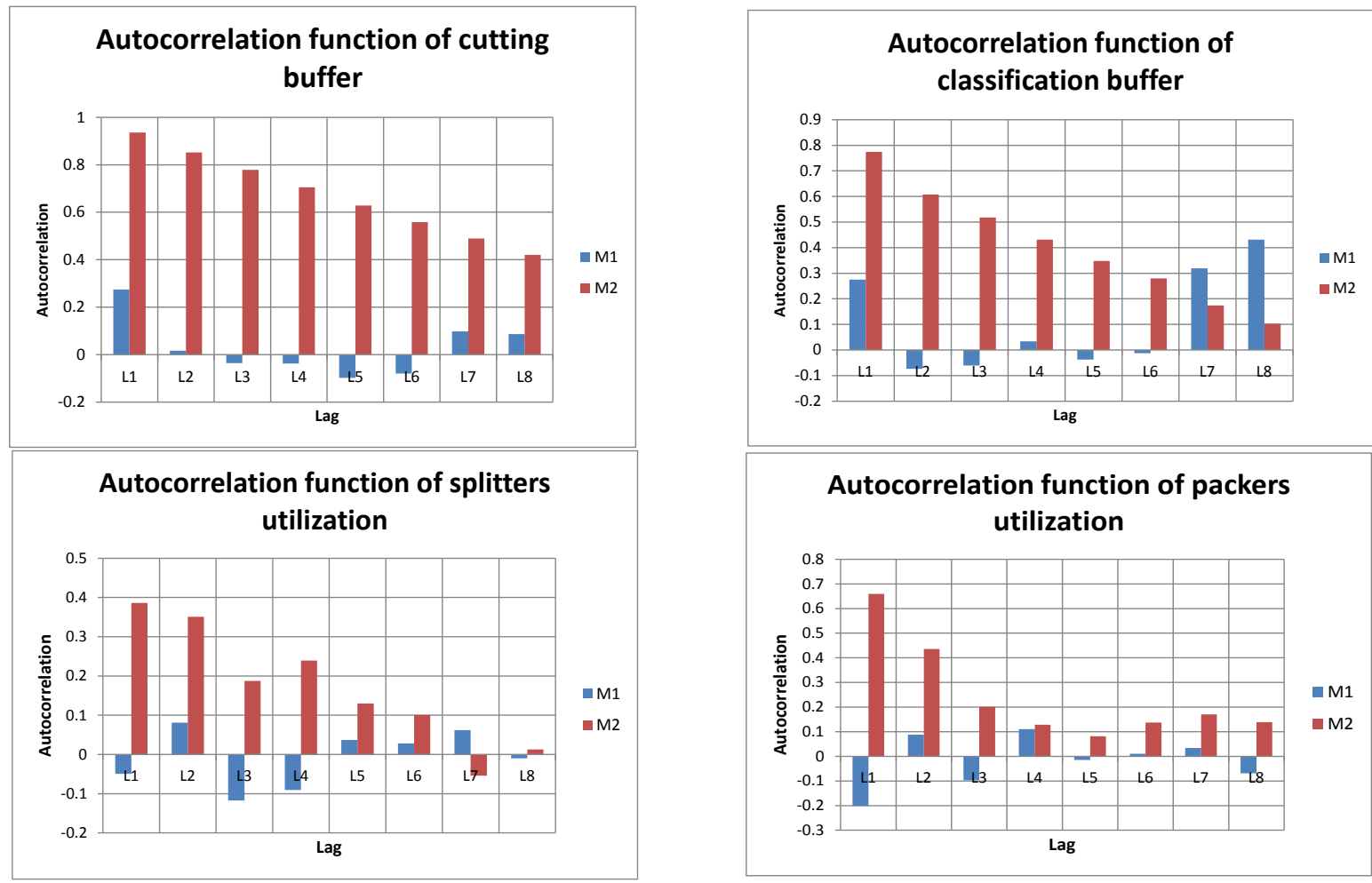

Figure 8. Autocorrelation functions for daily averages of buffer contents and resources.

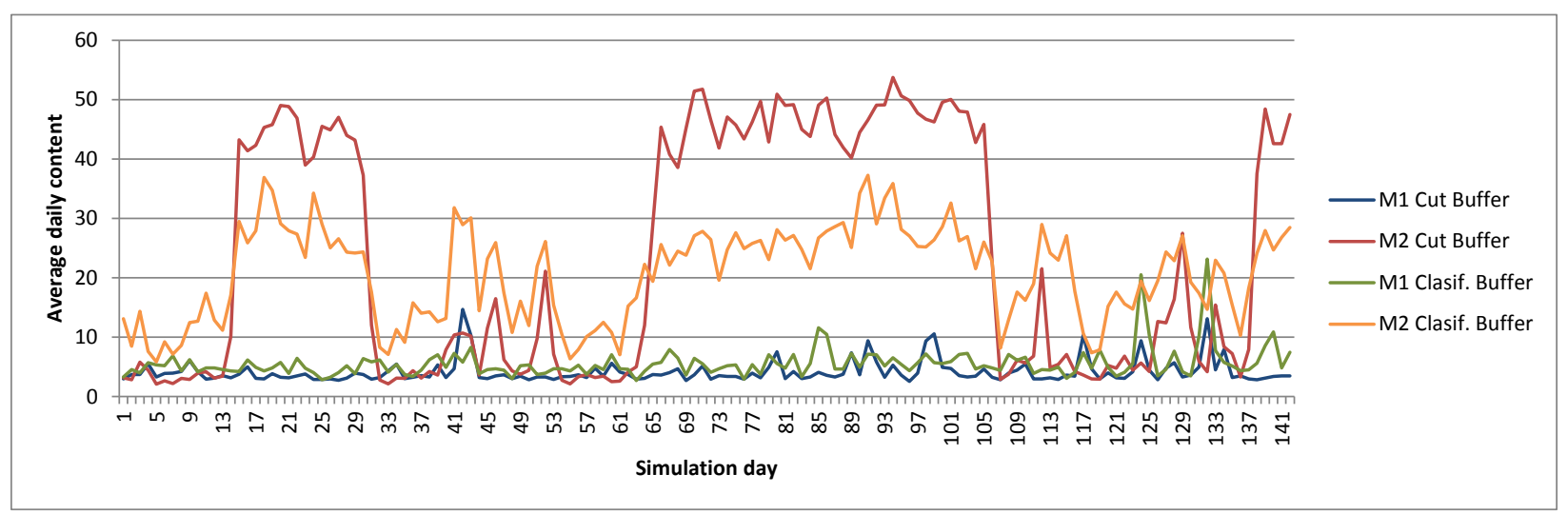

Figure 9. Time series of average daily content of buffers for models 1 and 2 (M1 and M2). 


\section{CONCLUSIONS}

Autocorrelation in manufacturing processes has been analyzed by means of three simulation experiments. In the first experiment, autocorrelation in cycle time series has been shown to have diverse effects in process performance depending on the autocorrelation structure being considered. This result supports previous results provided in the literature. Generally, process performance is enhanced by negative autocorrelation effects and reduced by positive autocorrelation. More complex autocorrelation patterns affect performance depending on buffer capacities.

The second experiment consisted of the analysis of autocorrelation present in process parameters varying at different time scales. In this case, negative effects in performance have also been found, becoming more acute as the considered time series step increases. In this case, both negative and positive autocorrelation diminish performance. However, for a disassembly process, autocorrelation in the product downstream routing probabilities do not seem to have a noticeable effect.

Finally, a real case study has been presented. This case provides a practical example on how considering autocorrelation may enhance the accuracy of a model. A model in which autocorrelation in process parameters is considered exhibits a more similar behavior to the real system. In addition, autocorrelation functions in key process variables such as daily buffers contents and resources utilization depict evident differences in this model.

\section{ACKNOWLEDGMENTS}

We wish to express our gratitude to the Xunta de Galicia, which has funded this work through the research project "Análise mediante simulación para a optimización do proceso de fabricación de lousas" (IN841C-2008/108). We also want to express our gratitude to the company Europizarras S.A. for their confidence and commitment.

\section{REFERENCES}

Altiok, T., \& Melamed, B. (2001). The case for modeling correlation in manufacturing systems. IEE Transactions, 33(9), 779-791.

Baines, T., Mason, S., Siebers, P.-O., \& Ladbrook, J. (2004). Humans: the missing link in manufacturing simulation? Simulation Modelling Practice and Theory, 12(7-8), 515-526. doi:10.1016/S1569190X(03)00094-7

Balcioglu, B., Jagerman, D. L., \& Altiok, T. (2007). Approximate mean waiting time in a GI / D /1 queue with autocorrelated times to failures. IIE Transactions, 39(10), 985-996. doi:10.1080/07408170701275343

Balcıglu, B., Jagerman, D. L., \& Altık, T. (2008). Merging and splitting autocorrelated arrival processes and impact on queueing performance. Performance Evaluation, 65(9), 653-669. doi:10.1016/j.peva.2008.02.003

Banks, J., Carson, J. S., Nelson, B. L., \& Nicol, D. M. (2010). Discrete-Event System Simulation (p. 640).

Crespo Pereira, D., del Rio Vilas, D., Rego Monteil, N., \& Rios Prado, R. (2012). Simulation and Highly Variable Environments: A Case Study in a Natural Roofing Slates Manufacturing Plant. Use Cases of Discrete Event Simulation (pp. 147-178).

Hejn Nielsen, E. (2007). Autocorrelation in queuing network-type production systems-Revisited. International Journal of Production Economics, 110(1-2), 138-146. doi:10.1016/j.ijpe.2007.02.014

Hendricks, K. B., \& Mcclain, J. O. (1993). The Output Processes of Serial Production Lines of General Machines with Finite Buffers. Management Science, 39(10), 1194-1201.

Livny, M., Melamed, B., \& Tsiolis, A. K. (1993). The Impact of Autocorrelation on Queuing Systems. Management Science, 39(3), 322-339.

Luxhoj, J. T., \& Shyur, H. (1995). Reliability curve fitting for aging helicopter components. Reliability Engineering \& System Safety, 48(3), 229-234. doi:10.1016/0951-8320(95)00018-W 
Melamed, B., \& Hill, J. R. (1995). A Survey of TES Modeling Applications. SIMULATION, 64(6), 353370. doi:10.1177/003754979506400602

Mertens, K., Vaesen, I., Löffel, J., Kemps, B., Kamers, B., Zoons, J., Darius, P., et al. (2009). An intelligent control chart for monitoring of autocorrelated egg production process data based on a synergistic control strategy. Computers and Electronics in Agriculture, 69(1), 100-111. doi:10.1016/j.compag.2009.07.012

Rego Monteil, N., del Rio Vilas, D., Crespo Pereira, D., \& Rios Prado, R. (2010). A Simulation-Based Ergonomic Evaluation for the Operational Improvement of the Slate Splitters Work. Proceedings of The 22nd European Modeling \& Simulation Symposium (pp. 191-200).

Schomig, A., \& Mittler, M. (1995). Autocorrelation of cycle times in semiconductor manufacturing systems. Proceedings of the 1995 Winter Simulation Conference (pp. 865-872).

Takahashi, K., Nakamura, N. (1998). The effect of autocorrelated demand in JIT production systems. International Journal of Production Research, 36(5), 1159-1176.

Timothy M. Young, \& Winistorfer, P. M. (2001). The effects of autocorrelation on real-time statistical process control with solutions for forest products manufacturers. Forest Products Journal, 51(11/12), 70-77.

del Rio Vilas, D., Crespo Pereira, D., Crespo Mariño, J. L., \& Garcia del Valle, A. (2009). Modelling and Simulation of a Natural Roofing Slates Manufacturing Plant. Proceedings of The International Workshop on Modelling and Applied Simulation (pp. 232-239).

\section{AUTHOR BIOGRAPHIES}

DIEGO CRESPO PEREIRA holds an MSc in Industrial Engineering from the UDC and he is currently studying for a $\mathrm{PhD}$. He is Assistant Professor of the Department of Economic Analysis and Company Management of the University of A Coruna. He also works in the GII as a research engineer since 2008. He is mainly involved in the development of $R \& D$ projects related to industrial and logistical processes optimization. His email address is diego.crespo@udc.es.

DAVID DEL RIO VILAS holds an MSc in Industrial Engineering. He is Adjunct Professor of the Department of Economic Analysis and Company Management of the UDC and research engineer in the GII of the UDC since 2007. Since 2010 he works as a R\&D Coordinator for two different privately held companies in the Civil Engineering sector. He is mainly involved in R\&D projects development related to industrial and logistical processes optimization. His email address is daviddelrio@udc.es.

NADIA REGO MONTEIL obtained her MSc in Industrial Engineering in 2010. She works as a research engineer at the Engineering Research Group (GII) of the University of A Coruna (UDC) where she is also studying for a PhD. Her areas of major interest are in the fields of Ergonomics, Process Optimization and Production Planning. Her email address is nadia.rego@udc.es.

ROSA RIOS PRADO works as a research engineer in the GII of the UDC since 2009. She holds an MSc in Industrial Engineering from the UDC and she is currently studying for a $\mathrm{PhD}$. She has previous professional experience as an Industrial Engineer in several installations engineering companies. She is mainly devoted to the development of transportation and logistical models for the assessment of multimodal networks and infrastructures by means of simulation techniques. Her email address is rrios@udc.es.

ALEJANDRO GARCIA DEL VALLE is an Industrial Engineer by the Polytechnic University of Madrid and holds a $\mathrm{PhD}$ in Industrial Engineering. He is Professor and one of the founders of the GII at the University of A Coruna. He has led many research projects about simulation and optimization of industrial, logistical and service systems. His email address is agvalle@udc.es. 